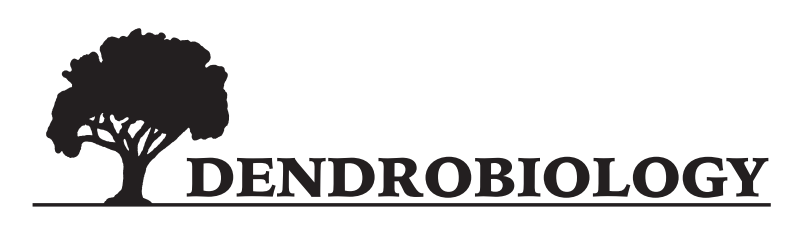

2015, vol. 73, 91-101

http://dx.doi.org/10.12657/denbio.073.009

\title{
Zuzana Špinlerová
}

\section{Determination of the biomass of Pinus mugo stands}

Received: 27 February 2014; Accepted: 29 August 2014

\begin{abstract}
The dwarf pine stands on unoriginal sites in mountainous areas of the Czech Republic are a current topic of scientific discussion. One of these sites is on the summits of the Hrubý Jeseník Mts. Various proposals for dwarf pine removal have been hindered by the absence of charts or tables that could be used to calculate how much biomass would need to be removed. Therefore, we created a methodology for dwarf pine biomass determination and applied it to five research transects of different ages. Based on the biomass estimates, we created trend curves illustrating the increase in biomass (dependent on age) as well as equations that could be used to roughly estimate the biomass of any dwarf pine stand, regardless of age or canopy level, for sites above the timberline in Hrubý Jeseník Mts. The equations for biomass calculations could also be applied to other mountain ranges where artificially planted dwarf pines of the same seed origin or the same morphological appearance as those existing in the Hrubý Jeseník Mts. are found.
\end{abstract}

Additional key words: dwarf pine, aboveground biomass, issue of indigeneity (originality problem), removal

Address: Z. Špinlerová, Mendel University in Brno, Faculty of Forestry and Wood Technology, Department of Forest Botany, Dendrology and Geobiocoenology, Zemědělská 3, 61300 Brno, Czech Republic, e-mail: zuzana.spinlerova@mendelu.cz

\section{Introduction}

Pinus mugo Turra /syn. P. mugo ssp. mughus (Scop.) Dom./ is a variable and taxonomically complicated species. Complex of this pine has many unknown traits, including the origin of the taxa, the classification and the natural range of Pinus mugo. The issues of its origin and the taxonomic classification of individual shrubs and populations have been addressed by many methods examining various properties of specimens or entire populations. Older methods include studying morphological and anatomical features, such as biometric analyses, but advances in technology favor newer polyprenol analyses, isoen- zyme genotype analyses and allozyme studies of genetic variability.

The presence of non-indigenous or probable non-indigenous stands in the Jeseníky, Orlické hory and Krkonoše mountains has become a topic of much debate in the Czech Republic. The dwarf pine is indigenous to the peat bogs of the supramontane belt of the Krkonoše Mts. and is also a natural component of many biotopes within the subalpine belt. However, introduced dwarf pines grown from seeds from Germany or Austria were planted in the ridges at the end of the $19^{\text {th }}$ century (Lokvenc 2003). These non-indigenous stands have recently been carefully and gradually removed. 
The non-indigeneity of the dwarf pine in the Hrubý Jeseník Mts. (part of the Jeseníky Mts.) has been discussed because these mountains contain more than 350 ha (Šenfeldr et al. 2012) of planted dwarf pine stands. These stands thrive, as they are typical mountain heliophytes with optimal growth design. The dwarf pine was first planted in the Hrubý Jeseník Mts. in 1877 in the Bruntál domain (because of shift of the timberline and water flow regulation) and the planting continued in 1921 after disastrous landslides in the Šumperk district. The seed for these plantings was purchased in Innsbruck or in Wiener Neustadt. Other, more extensive planting occurred in the 1970s in the area of Petrovy kameny - Velký Máj, including the cirques of Velká kotlina and Malá kotlina. Unfortunately, there are no records about the origin of seed (Holubičková 1980).

Due to the alleged influence of the dwarf pine on the gradual deterioration of the condition of several valuable eco-phenomena of the Hrubý Jeseník summits (e.g., disruption of arcto-alpine communities of tundra character), several proposals for dwarf pine reduction have been created and partially implemented by the Administration of the Protected Landscape Area of Jeseníky Mts.

In response to the dwarf pine reduction proposals (without solution of technical-institutional aspects of the problem), the primary aim of this study, funded by the GS LČR (Grant service of the Forests of the Czech Republic), is to estimate the aboveground biomass of the dwarf pines growing above the timberline in the Hrubý Jeseník Mts. that would have to be addressed before the potential removal of the stands. Because there are no tables that determine the biomass of the dwarf pine stands, the aims of this study were to create a methodology for determining the dwarf pine biomass and trend curves expressing the increase in biomass based on age. These tools were further used to derive equations for biomass estimation in all dwarf pine stands in Hrubý Jeseník Mts., of various age classes and various levels of canopy (meant as various canopy density).

\section{Overview of the literature on dwarf pine growth and the formation of biomass}

Authors have generally concentrated more on dwarf pine growth dynamics than on the biomass. There are essentially three categories of research:

1. Studies about the dynamics of dwarf pine stands using analyses of aerial photos.

These studies compare the current conditions with those shown in historical aerial photos and are analyses of the changes in the cover of dwarf pine stands over time. This topic has been addressed by many Czech and foreign authors using manual visual methods and automated classification methods, e.g., Fišerová (1991), Lokvenc and Vacek (1991), Carmel and Kadmon (1998), Kadmon and Harari-Kremer (1999), Potočka (1999), Souček et al. (2001), Halounová (2004), Müllerová (2005), Wild (2006a), Palombo et al. (2013) and others.

The dynamics of dwarf pine stands using analyses of aerial photos specifically in the Hrubý Jeseník Mts. have been addressed by Hošek et al. (2005), Wild (2006b), Wild et al. (2007).

2. Studies about the growth dynamics of dwarf pine stands using dendrometric and dendrochronological methods.

Analyses of the diameter increments of the dwarf pine have been conducted by Kolischuk and Berko (1967), Kolischuk (1969), Heikkinen (1980), Bitterli (1987), Corona (1987a), Simon and Drápela (1987) and Hohl et al. (2002), Kyncl and Wild (2004), Špinlerová and Martinková (2006, 2009), Palombo et al. (2014), among others.

Jeseníky dwarf pines on sites exposed to pollution were studied partially by Simon and Drápela (1987). Their results showed that the diameter increment had not fluctuated for thirty years. Dendrochronological analyses of selected shrubs in Hrubý Jeseník Mts. were performed by Hošek et al. (2005).

3. Studies about the growth dynamics of dwarf pines based on length increment.

The length increment of the dwarf pine has not been well monitored. An older study by Popovic (1976) describes the growth of the dwarf pine in the Vršič Mts. and a more recent study by Špinlerová and Martinková (2006) contains a growth analysis of the dwarf pine in the Orlické hory Mts. The length increment of the dwarf pine (specifically the length of the last ten increments of the main branches) in Jeseníky Mts. was studied by Hošek et al. (2005).

The knowledge gained from studies into the dynamics of stands provides a foundation for theories about the speed of biomass formation. Literature summarizing the data about the amounts of dwarf pine biomass (of single specimens or entire stands) is scarce. The authors more often address the biomass of the herbal (moss) growth under the dwarf pine - e.g., Kubíček et al. (1983), Kubíček (2001) or the total aboveground biomass of the community. Bliss (1962) provided a production of tundra ecosystems within one growing season and the productivity of the natural tundra communities was evaluated by Malinovskij (1984) and Archibold et al. (1995). The volume and weight of the "stems" and branches and the weight of needles within one dwarf pine specimen growing at an altitude of $1900 \mathrm{~m}$ in the Vršič Mts. was published by Popovic (1976). The range 
of the determined volume of dwarf pine stems from eastern Trentino (Northern Italy) presented Corona (1987b).

\section{Methods}

The original intention - to determine the biomass of one specimen or the polycormon of one mother plant - was abandoned after the terrain survey. The dwarf pines in the area grow wildly with a complicated vegetative propagation, which makes terrain orientation impossible. Therefore, five representative $100 \%$ closed-canopy transects of non-indigenous stands (with areas of $4 \mathrm{~m}^{2}$ ) of different ages or dwarf pine heights (stands of the $15^{\text {th }}, 8^{\text {th }}, 4^{\text {th }}$ and $2^{\text {nd }}$ age class) were selected on sites near the summit of Keprník (K) and in the Větrná louka (V) /Fig. 1, Table $1 /$. (One age class in the Czech forestry terminology in this case means age range of 10 years. For example, the stand of the $2^{\text {nd }}$ age class may have 11 to 20 years.) All transects were located in the Protected Landscape Area, transects $\mathrm{K}$ also in the National Nature Reserve (NNR) Šerák - Keprník and V in the NNR Praděd.

The dwarf pine is, as mentioned, in all selected transects difficult growing, generating polycormons. Shrubs are densely branched, closed. The main branches (plumules) of the shrubs are loosing terminal character (the apical dominance); reaching almost the same height that does not exceed $3.5 \mathrm{~m}$. The branches are prostrate at the base, but then bent into an arc and grow into an upright form (form is close to cubic paraboloid).

The understory in these stands (transects) is very poor, only with a few species, as Vaccinium myrtillus, Avenella flexuosa, Calamagrostis villosa, Nardus stricta, Trientalis europaea or Calluna vulgaris.

All aboveground dwarf pine biomass was removed from the transects.

The material was transported to the laboratory, where the fresh mass and volume were measured. The masses of the large timber (the above-ground woody mass including bark with a diameter greater than $0.07 \mathrm{~m}$ ), small timber (the above-ground woody mass including bark with a diameter less than 0.07 $\mathrm{m})$ and needles were determined separately using a hanging scale (digital, $100 \mathrm{~g}$ accuracy). The mass of cones was determined using a electronic scales Kern 822 (1000 g accuracy). The volume of the large tim-

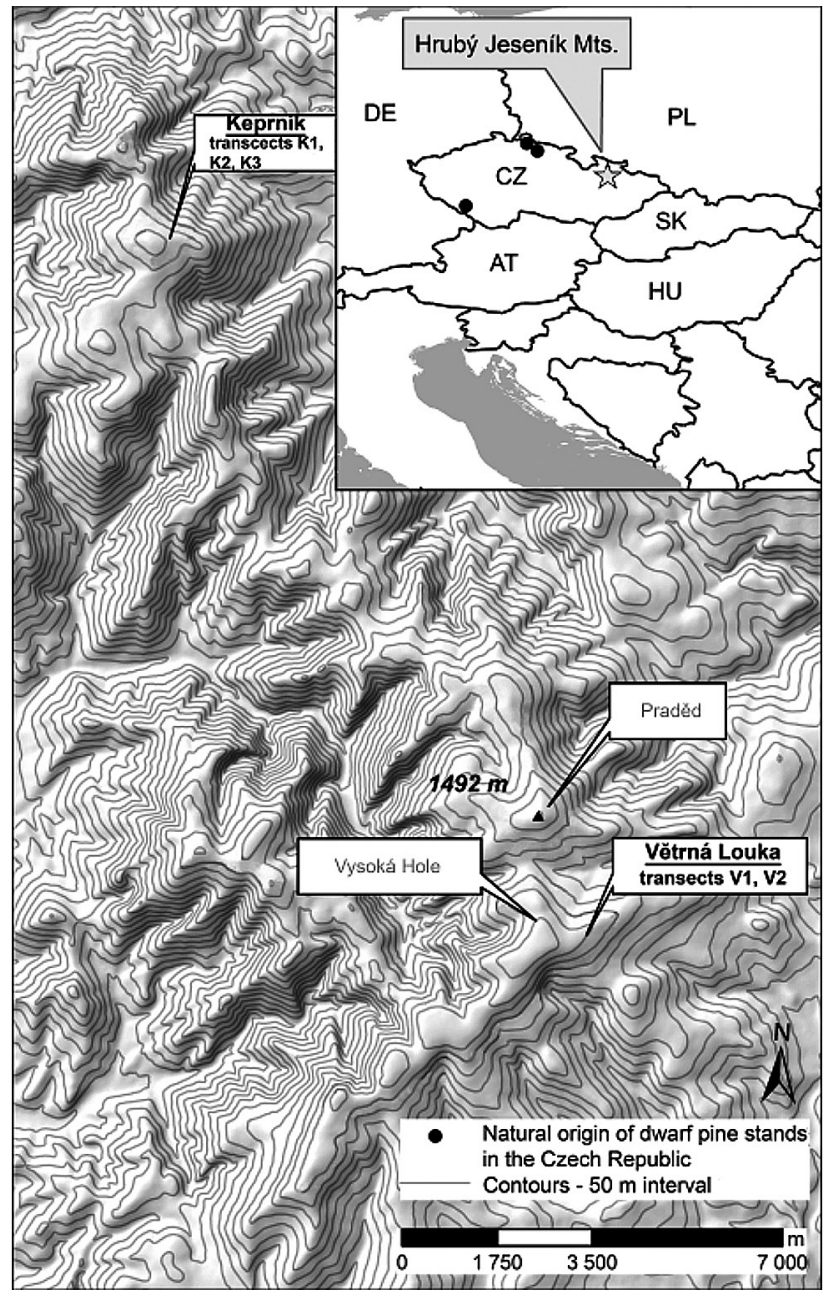

Fig. 1. The overall study area with location of representative transects $\mathrm{K} 1, \mathrm{~K} 2, \mathrm{~K} 3$ and V1, V2. All dwarf pine stands in Hrubý Jeseník Mts. are non-indigenous. Natural stands in the Czech Republic are relatively distant, as also natural stands in Poland or in Slovakia

ber was calculated as the sum of volumes of $25 \mathrm{~cm}$ sections using a formula for the calculation of a truncated cone (Packová and Maděra 2004).

Truncated cone calculation formula:

$$
V=\frac{\pi v}{3}\left(r_{1}^{2}+r_{1} r_{2}+r_{2}^{2}\right)
$$

where $r_{1}$ and $r_{2}$ are the radii of the bases; $v$ is the height.

The volume of the small timber and needles was calculated from the determined fresh mass and vol-

Table 1. Location of the selected transects

\begin{tabular}{lcr}
\multicolumn{1}{c}{ Site } & Transect & Age class \\
Keprník & K1 & 15 (150 years) \\
Keprník & K2 & 15 (150 years) \\
Keprník & K3 & 8 (80 years) \\
Větrná louka & V1 & 4 (39 years) \\
Větrná louka & V2 & 2 (18 years)
\end{tabular}

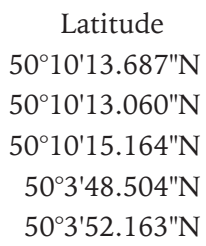

Latitude
$50^{\circ} 10^{\prime} 13.687 " \mathrm{~N}$
$50^{\circ} 10^{\prime} 13.060^{\prime \prime} \mathrm{N}$
$50^{\circ} 10^{\prime} 15.164^{\prime \prime} \mathrm{N}$
$50^{\circ} 3^{\prime} 48.504^{\prime \prime} \mathrm{N}$
$50^{\circ} 3^{\prime} 52.163^{\prime \prime} \mathrm{N}$

Longitude

$17^{\circ} 7^{\prime} 1.709 " \mathrm{E}$

$17^{\circ} 7^{\prime} 2.387^{\prime \prime} \mathrm{E}$

$17^{\circ} 7^{\prime} 1.963$ "E

$17^{\circ} 14^{\prime} 31.664^{\prime \prime} \mathrm{E}$

$17^{\circ} 14^{\prime} 30.024^{\prime \prime} \mathrm{E}$ 


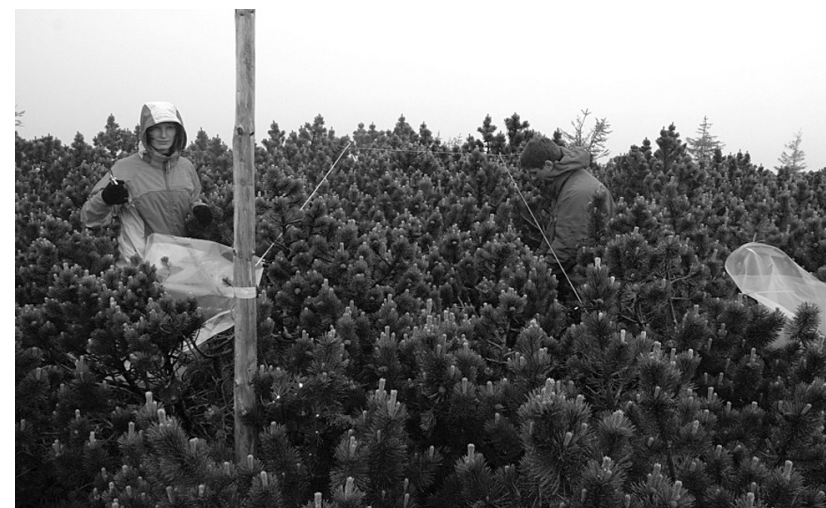

Fig. 2. Transect K3 - 80-year-old stand (the stand of the $8^{\text {th }}$ age class) in Keprník (Photo: Z. Špinlerová)

ume of ten sample branches and fifty couples of needles in the graduated cylinder.

Next, the material was dried at $105^{\circ} \mathrm{C}$ in the drying chamber to a constant weight and the dry mass was determined (using the digital hanging scale, for cones using electronic scales). Samples of needles (10 couples from each year) were scanned both before and after drying and their area was determined using the Quick PHOTO MICRO application (developed by Promicra, s.r.o.; Prague; Czech Republic; Europe). The area of the sample needles was averaged for each year and the average was used to calculate the total area of the needles (the needle surface area calculated from the averaged surface area of needles and the total number of needles occurring in the area of $4 \mathrm{~m}^{2}$ ).

All of the results were converted to an area of 1 $\mathrm{m}^{2}$, or 1 ha. The values for the two 150-year-old plots were averaged.

Based on the results from the transects, trend curves expressing the increase in fresh biomass (mass and volume) in relation to age were created; further, the trend curves were used to derive equations for the biomass calculation for dwarf pine stands of different age classes.

The equations were used to express the biomass of all dwarf pine stands with different canopy levels on sites above the timberline of the Hrubý Jeseník Mts.

\section{Results}

Figure 2 shows the process of setting out the selected plots.

The results for specific transects are presented in Table 2. The results converted to an area of $1 \mathrm{~m}^{2}$, are in Table 3. The parameters were determined separately for large timber, small timber, needles and

Table 2. Parameters determined in selected transects

\begin{tabular}{|c|c|c|c|c|c|c|c|c|c|}
\hline \multicolumn{2}{|c|}{ Transect K1 $(\approx 150 \mathrm{yr})$} & \multicolumn{2}{|c|}{ Transect K2 $(\approx 150 \mathrm{yr})$} & \multicolumn{2}{|c|}{ Transect K3 $(\approx 80 \mathrm{yr})$} & \multicolumn{2}{|c|}{ Transect V1 $(\approx 40 \mathrm{yr})$} & \multicolumn{2}{|c|}{ Transect V2 $(\approx 20 \mathrm{yr})$} \\
\hline \multicolumn{2}{|c|}{ Height $\approx 2.5 \mathrm{~m}$} & \multicolumn{2}{|c|}{ Height $\approx 3.2 \mathrm{~m}$} & \multicolumn{2}{|c|}{ Height $\approx 1.3 \mathrm{~m}$} & \multicolumn{2}{|c|}{ Height $\approx 1.3 \mathrm{~m}$} & \multicolumn{2}{|c|}{ Height $\approx 1.2 \mathrm{~m}$} \\
\hline \multicolumn{2}{|c|}{ Fresh mass $/ 4 \mathrm{~m}^{2}$} & \multicolumn{2}{|c|}{ Fresh mass $/ 4 \mathrm{~m}^{2}$} & \multicolumn{2}{|c|}{ Fresh mass $/ 4 \mathrm{~m}^{2}$} & \multicolumn{2}{|c|}{ Fresh mass $/ 4 \mathrm{~m}^{2}$} & \multicolumn{2}{|c|}{ Fresh mass $/ 4 \mathrm{~m}^{2}$} \\
\hline $\begin{array}{l}\text { Large } \\
\text { timber }\end{array}$ & $60 \mathrm{~kg}$ & $\begin{array}{l}\text { Large } \\
\text { timber }\end{array}$ & $93.3 \mathrm{~kg}$ & $\begin{array}{l}\text { Large } \\
\text { timber }\end{array}$ & $10.0 \mathrm{~kg}$ & $\begin{array}{l}\text { Large } \\
\text { timber }\end{array}$ & $2.6 \mathrm{~kg}$ & $\begin{array}{l}\text { Large } \\
\text { timber }\end{array}$ & $1.3 \mathrm{~kg}$ \\
\hline $\begin{array}{l}\text { Small } \\
\text { timber }\end{array}$ & $33.5 \mathrm{~kg}$ & $\begin{array}{l}\text { Small } \\
\text { timber }\end{array}$ & $5.1 \mathrm{~kg}$ & $\begin{array}{l}\text { Small } \\
\text { timber }\end{array}$ & $50.1 \mathrm{~kg}$ & $\begin{array}{l}\text { Small } \\
\text { timber }\end{array}$ & $42.9 \mathrm{~kg}$ & $\begin{array}{l}\text { Small } \\
\text { timber }\end{array}$ & $39.2 \mathrm{~kg}$ \\
\hline Needles & $5.8 \mathrm{~kg}$ & Needles & $14.5 \mathrm{~kg}$ & Needles & $12.2 \mathrm{~kg}$ & Needles & $13.4 \mathrm{~kg}$ & Needles & $13.5 \mathrm{~kg}$ \\
\hline Cones & $0.8 \mathrm{~kg}$ & Cones & $0.8 \mathrm{~kg}$ & Cones & $1.0 \mathrm{~kg}$ & Cones & $0.488 \mathrm{~kg}$ & Cones & $0.130 \mathrm{~kg}$ \\
\hline Total & $100.1 \mathrm{~kg}$ & Total & $113.7 \mathrm{~kg}$ & Total & $73.3 \mathrm{~kg}$ & Total & $59.388 \mathrm{~kg}$ & Total & $54.13 \mathrm{~kg}$ \\
\hline \multicolumn{2}{|c|}{ Dry mass $/ 4 \mathrm{~m}^{2}$} & \multicolumn{2}{|c|}{ Dry mass $/ 4 \mathrm{~m}^{2}$} & \multicolumn{2}{|c|}{ Dry mass $/ 4 \mathrm{~m}^{2}$} & \multicolumn{2}{|c|}{ Dry mass $/ 4 \mathrm{~m}^{2}$} & \multicolumn{2}{|c|}{ Dry mass $/ 4 \mathrm{~m}^{2}$} \\
\hline $\begin{array}{l}\text { Large } \\
\text { timber }\end{array}$ & $40.6 \mathrm{~kg}$ & $\begin{array}{l}\text { Large } \\
\text { timber }\end{array}$ & $63.8 \mathrm{~kg}$ & $\begin{array}{l}\text { Large } \\
\text { timber }\end{array}$ & $6.8 \mathrm{~kg}$ & $\begin{array}{l}\text { Large } \\
\text { timber }\end{array}$ & $1.6 \mathrm{~kg}$ & $\begin{array}{l}\text { Large } \\
\text { timber }\end{array}$ & $0.6 \mathrm{~kg}$ \\
\hline $\begin{array}{l}\text { Small } \\
\text { timber }\end{array}$ & $22.2 \mathrm{~kg}$ & $\begin{array}{l}\text { Small } \\
\text { timber }\end{array}$ & $2.3 \mathrm{~kg}$ & $\begin{array}{l}\text { Small } \\
\text { timber }\end{array}$ & $34.1 \mathrm{~kg}$ & $\begin{array}{l}\text { Small } \\
\text { timber }\end{array}$ & $25.3 \mathrm{~kg}$ & $\begin{array}{l}\text { Small } \\
\text { timber }\end{array}$ & $19.2 \mathrm{~kg}$ \\
\hline Needles & $2.8 \mathrm{~kg}$ & Needles & $6.5 \mathrm{~kg}$ & Needles & $6.5 \mathrm{~kg}$ & Needles & $6.4 \mathrm{~kg}$ & Needles & $6.8 \mathrm{~kg}$ \\
\hline Cones & $0.4 \mathrm{~kg}$ & Cones & $0.420 \mathrm{~kg}$ & Cones & $0.532 \mathrm{~kg}$ & Cones & $0.242 \mathrm{~kg}$ & Cones & $0.065 \mathrm{~kg}$ \\
\hline Total & $66 \mathrm{~kg}$ & Total & $73.02 \mathrm{~kg}$ & Total & $47.932 \mathrm{~kg}$ & Total & $33.542 \mathrm{~kg}$ & Total & $26.665 \mathrm{~kg}$ \\
\hline \multicolumn{2}{|c|}{ Fresh mass volume $/ 4 \mathrm{~m}^{2}$} & \multicolumn{2}{|c|}{ Fresh mass volume $/ 4 \mathrm{~m}^{2}$} & \multicolumn{2}{|c|}{ Fresh mass volume $/ 4 \mathrm{~m}^{2}$} & \multicolumn{2}{|c|}{ Fresh mass volume $/ 4 \mathrm{~m}^{2}$} & \multicolumn{2}{|c|}{ Fresh mass volume $/ 4 \mathrm{~m}^{2}$} \\
\hline $\begin{array}{l}\text { Large } \\
\text { timber }\end{array}$ & $0.08 \mathrm{~m}^{3}$ & $\begin{array}{l}\text { Large } \\
\text { timber }\end{array}$ & $0.12 \mathrm{~m}^{3}$ & $\begin{array}{l}\text { Large } \\
\text { timber }\end{array}$ & $0.0284 \mathrm{~m}^{3}$ & $\begin{array}{l}\text { Large } \\
\text { timber }\end{array}$ & $0.0072 \mathrm{~m}^{3}$ & $\begin{array}{l}\text { Large } \\
\text { timber }\end{array}$ & $0.0024 \mathrm{~m}^{3}$ \\
\hline $\begin{array}{l}\text { Small } \\
\text { timber }\end{array}$ & $0.04 \mathrm{~m}^{3}$ & $\begin{array}{l}\text { Small } \\
\text { timber }\end{array}$ & $0.01 \mathrm{~m}^{3}$ & $\begin{array}{l}\text { Small } \\
\text { timber }\end{array}$ & $0.1036 \mathrm{~m}^{3}$ & $\begin{array}{l}\text { Small } \\
\text { timber }\end{array}$ & $0.0776 \mathrm{~m}^{3}$ & $\begin{array}{l}\text { Small } \\
\text { timber }\end{array}$ & $0.0612 \mathrm{~m}^{3}$ \\
\hline Needles & $0.0444 \mathrm{~m}^{3}$ & Needles & $0.0912 \mathrm{~m}^{3}$ & Needles & $0.0544 \mathrm{~m}^{3}$ & Needles & $0.0704 \mathrm{~m}^{3}$ & Needles & $0.0664 \mathrm{~m}^{3}$ \\
\hline Total & $0.1644 \mathrm{~m}^{3}$ & Total & $0.2212 \mathrm{~m}^{3}$ & Total & $0.1864 \mathrm{~m}^{3}$ & Total & $0.1552 \mathrm{~m}^{3}$ & Total & $0.1300 \mathrm{~m}^{3}$ \\
\hline \multicolumn{2}{|c|}{ Needle area $/ 4 \mathrm{~m}^{2}$} & \multicolumn{2}{|c|}{ Needle area $/ 4 \mathrm{~m}^{2}$} & \multicolumn{2}{|c|}{ Needle area $/ 4 \mathrm{~m}^{2}$} & \multicolumn{2}{|c|}{ Needle area $/ 4 \mathrm{~m}^{2}$} & \multicolumn{2}{|c|}{ Needle area $/ 4 \mathrm{~m}^{2}$} \\
\hline Fresh & $8.36 \mathrm{~m}^{2}$ & Fresh & $20.52 \mathrm{~m}^{2}$ & Fresh & $21.64 \mathrm{~m}^{2}$ & Fresh & $20.64 \mathrm{~m}^{2}$ & Fresh & $20.44 \mathrm{~m}^{2}$ \\
\hline Dry & $7.56 \mathrm{~m}^{2}$ & Dry & $17.96 \mathrm{~m}^{2}$ & Dry & $17.72 \mathrm{~m}^{2}$ & Dry & $17.52 \mathrm{~m}^{2}$ & Dry & $16.24 \mathrm{~m}^{2}$ \\
\hline \multicolumn{2}{|c|}{$\begin{array}{c}\text { Number of needle } \\
\text { pairs } / 4 \mathrm{~m}^{2}\end{array}$} & \multicolumn{2}{|c|}{$\begin{array}{l}\text { Number of needle } \\
\text { pairs } / 4 \mathrm{~m}^{2}\end{array}$} & $\begin{array}{r}\text { Numb } \\
\text { pa }\end{array}$ & $\begin{array}{l}\text { f needle } \\
4 \mathrm{~m}^{2}\end{array}$ & $\begin{array}{r}\text { Num } \\
p\end{array}$ & $\begin{array}{l}4 \mathrm{~m}^{2} \\
\end{array}$ & & $\begin{array}{l}\text { f needle } \\
4 \mathrm{~m}^{2}\end{array}$ \\
\hline & 9 pcs & 21 & 0 pcs & & 3 pcs & & 0 pcs & & 0 pcs \\
\hline
\end{tabular}




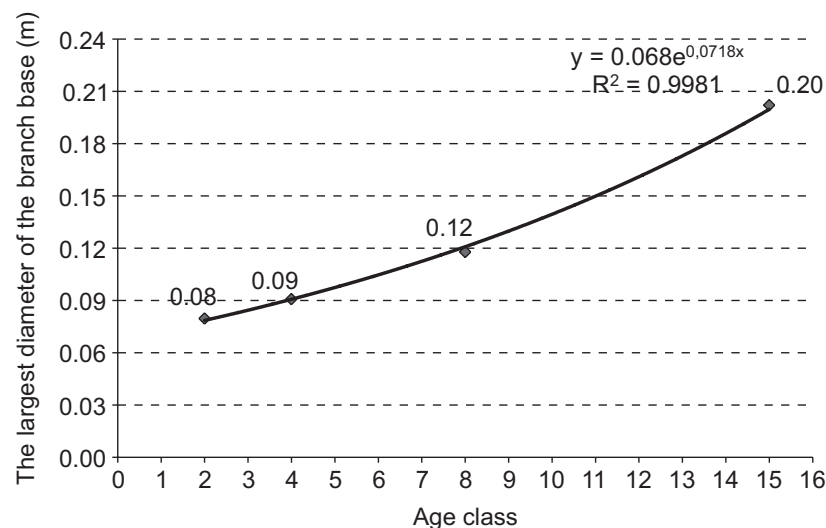

Fig. 3. Correlation between age class and the largest diameter of the branch base in selected transects (One age class means age range of 10 years. All dwarf pine ages in selected transects were at /or were approaching/ the upper limit of the age class)

cones. The total values were converted to an area of 1 ha (Table 4).

Figure 3 demonstrates correlation between the age class and the largest stem diameter of the branch base in selected transects. Figs 4 and 5 show the trend curves expressing the increasing biomass (mass and volume) based on age. The curves were used to create equations for the biomass calculation of dwarf pine stands of various age classes and canopy levels.

The equation for the calculation of the total fresh mass ( $\mathrm{t} / \mathrm{ha})$ :

$$
y=121.37 e^{0.0052 x}
$$

The equation for the calculation of the large timber fresh mass (t/ha):

$$
y=1.8395 e^{0.0313 x}
$$

in both equations: $\mathrm{x}=$ stand age.

The equation for the calculation of the total fresh mass volume ( $\left.\mathrm{m}^{3} / \mathrm{ha}\right)$ :

$$
y=81.838 \operatorname{Ln}(x)+86.386
$$

The equation for the calculation of the large timber fresh mass volume $\left(\mathrm{m}^{3} / \mathrm{ha}\right)$ :

$$
y=0.0113 x^{2}-0.0504 x+2.4725
$$

in both equations: $\mathrm{x}=$ stand age.

Using these equations and the data regarding the distribution and cover of the dwarf pine, we estimated the biomass above the timberline of the Hruby Jeseník Mts. The calculation shows that in the 360 ha area of the stands (of which 142 ha actually con-

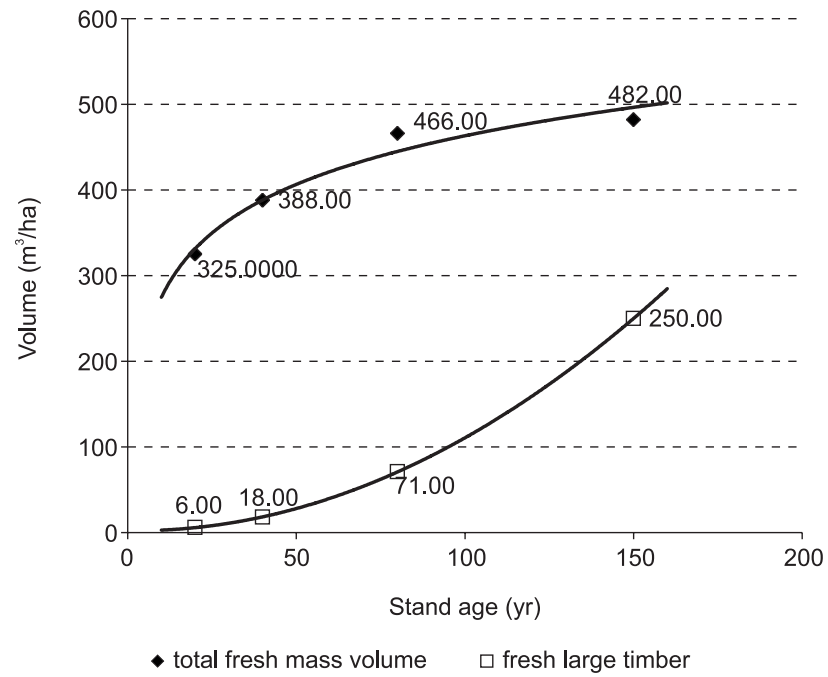

Fig. 5. Estimate of trends in the difference of volume $\left(\mathrm{m}^{3} /\right.$ ha) of the aboveground dwarf pine biomass, dependent on increasing age

tain dwarf pines), there is $30171 \mathrm{t}$ and $66325 \mathrm{~m}^{3}$ of biomass, of which $9892 \mathrm{t}$ and $18428 \mathrm{~m}^{3}$ is large timber. The specific values calculated for individual segments of the dwarf pine stands, as defined by Šenfeldr et al. (2013), are presented in Table 5.

\section{Discussion}

Due to the scarcity of literature on the biomass of the dwarf pine, it is difficult to compare our results with those of other authors. Previously published information about dwarf pine biomass either relates to individual specimens (Popovic 1976) or individual branches (Špinlerová and Martinková 2006). Theoretically, it would be possible to calculate or estimate the number of dwarf pine specimens in the explored

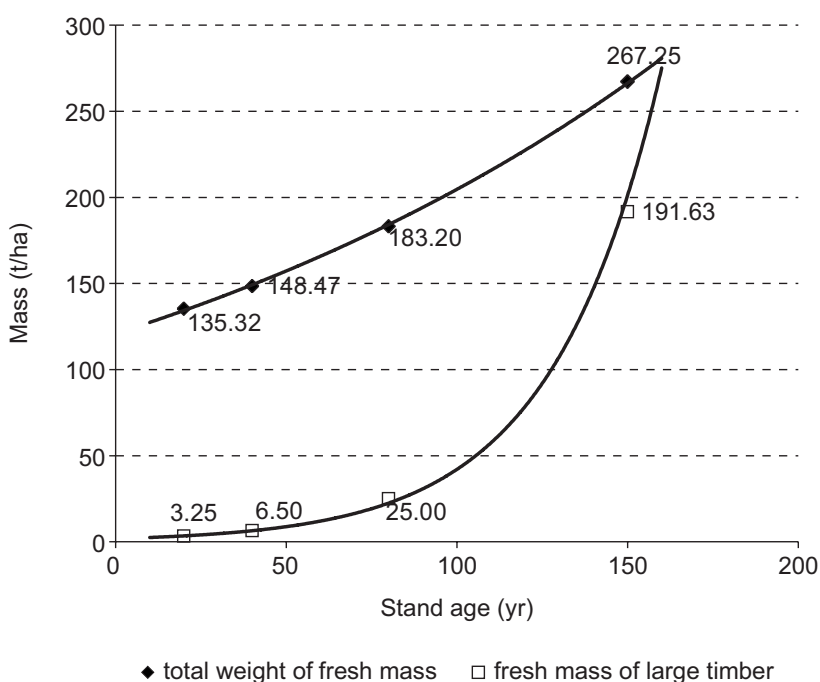

Fig. 4. Estimate of trends in the difference of mass ( $t / h a)$ of aboveground dwarf pine biomass, dependent on increasing age 
Table 3. Parameters determined in selected transects converted to an area of $1 \mathrm{~m}^{2}$

\begin{tabular}{|c|c|c|c|c|c|c|c|c|c|}
\hline \multicolumn{2}{|c|}{ Transect K1 $(\approx 150 \mathrm{yr})$} & \multicolumn{2}{|c|}{ Transect K2 $(\approx 150 \mathrm{yr})$} & \multicolumn{2}{|c|}{ Transect K3 $(\approx 80 \mathrm{yr})$} & \multicolumn{2}{|c|}{ Transect V1 $(\approx 40 \mathrm{yr})$} & \multicolumn{2}{|c|}{ Transect V2 $(\approx 20 \mathrm{yr})$} \\
\hline \multicolumn{2}{|c|}{ Height $\approx 2.5 \mathrm{~m}$} & \multicolumn{2}{|c|}{ Height $\approx 3.2 \mathrm{~m}$} & \multicolumn{2}{|c|}{ Height $\approx 1.3 \mathrm{~m}$} & \multicolumn{2}{|c|}{ Height $\approx 1.3 \mathrm{~m}$} & \multicolumn{2}{|c|}{ Height $\approx 1.2 \mathrm{~m}$} \\
\hline \multicolumn{2}{|c|}{ Fresh mass $/ \mathrm{m}^{2}$} & \multicolumn{2}{|c|}{ Fresh mass $/ \mathrm{m}^{2}$} & \multicolumn{2}{|c|}{ Fresh mass $/ \mathrm{m}^{2}$} & \multicolumn{2}{|c|}{ Fresh mass $/ \mathrm{m}^{2}$} & \multicolumn{2}{|c|}{ Fresh mass $/ \mathrm{m}^{2}$} \\
\hline $\begin{array}{l}\text { Large } \\
\text { timber }\end{array}$ & $15.000 \mathrm{~kg}$ & $\begin{array}{l}\text { Large } \\
\text { timber }\end{array}$ & $23.325 \mathrm{~kg}$ & $\begin{array}{l}\text { Large } \\
\text { timber }\end{array}$ & $2.5000 \mathrm{~kg}$ & $\begin{array}{l}\text { Large } \\
\text { timber }\end{array}$ & $0.6500 \mathrm{~kg}$ & $\begin{array}{l}\text { Large } \\
\text { timber }\end{array}$ & $0.3250 \mathrm{~kg}$ \\
\hline $\begin{array}{l}\text { Small } \\
\text { timber }\end{array}$ & $8.375 \mathrm{~kg}$ & $\begin{array}{l}\text { Small } \\
\text { timber }\end{array}$ & $1.275 \mathrm{~kg}$ & $\begin{array}{l}\text { Small } \\
\text { timber }\end{array}$ & $12.5250 \mathrm{~kg}$ & $\begin{array}{l}\text { Small } \\
\text { timber }\end{array}$ & $10.7250 \mathrm{~kg}$ & $\begin{array}{l}\text { Small } \\
\text { timber }\end{array}$ & $9.8000 \mathrm{~kg}$ \\
\hline Needles & $1.450 \mathrm{~kg}$ & Needles & $3.625 \mathrm{~kg}$ & Needles & $3.0500 \mathrm{~kg}$ & Needles & $3.3500 \mathrm{~kg}$ & Needles & $3.3750 \mathrm{~kg}$ \\
\hline Cones & $0.2 \mathrm{~kg}$ & Cones & $0.2 \mathrm{~kg}$ & Cones & $0.2500 \mathrm{~kg}$ & Cones & $0.1220 \mathrm{~kg}$ & Cones & $0.0325 \mathrm{~kg}$ \\
\hline Total & $25.025 \mathrm{~kg}$ & Total & $28.425 \mathrm{~kg}$ & Total & $18.3250 \mathrm{~kg}$ & Total & $14.8470 \mathrm{~kg}$ & Total & $13.5325 \mathrm{~kg}$ \\
\hline \multicolumn{2}{|c|}{ Dry mass $/ \mathrm{m}^{2}$} & \multicolumn{2}{|c|}{ Dry mass $/ \mathrm{m}^{2}$} & \multicolumn{2}{|c|}{ Dry mass $/ \mathrm{m}^{2}$} & \multicolumn{2}{|c|}{ Dry mass $/ \mathrm{m}^{2}$} & \multicolumn{2}{|c|}{ Dry mass $/ \mathrm{m}^{2}$} \\
\hline $\begin{array}{l}\text { Large } \\
\text { timber }\end{array}$ & $10.15 \mathrm{~kg}$ & $\begin{array}{l}\text { Large } \\
\text { timber }\end{array}$ & $15.95 \mathrm{~kg}$ & $\begin{array}{l}\text { Large } \\
\text { timber }\end{array}$ & $1.7000 \mathrm{~kg}$ & $\begin{array}{l}\text { Large } \\
\text { timber }\end{array}$ & $0.4000 \mathrm{~kg}$ & $\begin{array}{l}\text { Large } \\
\text { timber }\end{array}$ & $0.1500 \mathrm{~kg}$ \\
\hline $\begin{array}{l}\text { Small } \\
\text { timber }\end{array}$ & $5.550 \mathrm{~kg}$ & $\begin{array}{l}\text { Small } \\
\text { timber }\end{array}$ & $0.575 \mathrm{~kg}$ & $\begin{array}{l}\text { Small } \\
\text { timber }\end{array}$ & $8.5250 \mathrm{~kg}$ & $\begin{array}{l}\text { Small } \\
\text { timber }\end{array}$ & $6.3250 \mathrm{~kg}$ & $\begin{array}{l}\text { Small } \\
\text { timber }\end{array}$ & $4.8000 \mathrm{~kg}$ \\
\hline Needles & $0.700 \mathrm{~kg}$ & Needles & $1.625 \mathrm{~kg}$ & Needles & $1.6250 \mathrm{~kg}$ & Needles & $1.6000 \mathrm{~kg}$ & Needles & $1.7000 \mathrm{~kg}$ \\
\hline Cones & $0.100 \mathrm{~kg}$ & Cones & $0.105 \mathrm{~kg}$ & Cones & $0.1330 \mathrm{~kg}$ & Cones & $0.0605 \mathrm{~kg}$ & Cones & $0.0162 \mathrm{~kg}$ \\
\hline Total & $16.500 \mathrm{~kg}$ & Total & $18.255 \mathrm{~kg}$ & Total & $11.9830 \mathrm{~kg}$ & Total & $8.3855 \mathrm{~kg}$ & Total & $6.6662 \mathrm{~kg}$ \\
\hline \multicolumn{2}{|c|}{ Fresh mass volume $/ \mathrm{m}^{2}$} & \multicolumn{2}{|c|}{ Fresh mass volume $/ \mathrm{m}^{2}$} & \multicolumn{2}{|c|}{ Fresh mass volume $/ \mathrm{m}^{2}$} & \multicolumn{2}{|c|}{ Fresh mass volume $/ \mathrm{m}^{2}$} & \multicolumn{2}{|c|}{ Fresh mass volume $/ \mathrm{m}^{2}$} \\
\hline $\begin{array}{l}\text { Large } \\
\text { timber }\end{array}$ & $0.02 \mathrm{~m}^{3}$ & $\begin{array}{l}\text { Large } \\
\text { timber }\end{array}$ & $0.03 \mathrm{~m}^{3}$ & $\begin{array}{l}\text { Large } \\
\text { timber }\end{array}$ & $0.0071 \mathrm{~m}^{3}$ & $\begin{array}{l}\text { Large } \\
\text { timber }\end{array}$ & $0.0018 \mathrm{~m}^{3}$ & $\begin{array}{l}\text { Large } \\
\text { timber }\end{array}$ & $0.0006 \mathrm{~m}^{3}$ \\
\hline $\begin{array}{l}\text { Small } \\
\text { timber }\end{array}$ & $0.0100 \mathrm{~m}^{3}$ & $\begin{array}{l}\text { Small } \\
\text { timber }\end{array}$ & $0.0025 \mathrm{~m}^{3}$ & $\begin{array}{l}\text { Small } \\
\text { timber }\end{array}$ & $0.0259 \mathrm{~m}^{3}$ & $\begin{array}{l}\text { Small } \\
\text { timber }\end{array}$ & $0.0194 \mathrm{~m}^{3}$ & $\begin{array}{l}\text { Small } \\
\text { timber }\end{array}$ & $0.0153 \mathrm{~m}^{3}$ \\
\hline Needles & $0.0111 \mathrm{~m}^{3}$ & Needles & $0.0228 \mathrm{~m}^{3}$ & Needles & $0.0136 \mathrm{~m}^{3}$ & Needles & $0.0176 \mathrm{~m}^{3}$ & Needles & $0.0166 \mathrm{~m}^{3}$ \\
\hline Total & $0.0411 \mathrm{~m}^{3}$ & Total & $0.0553 \mathrm{~m}^{3}$ & Total & $0.0466 \mathrm{~m}^{3}$ & Total & $0.0388 \mathrm{~m}^{3}$ & Total & $0.0325 \mathrm{~m}^{3}$ \\
\hline \multicolumn{2}{|c|}{ Needle area $/ \mathrm{m}^{2}$} & \multicolumn{2}{|c|}{ Needle area $/ \mathrm{m}^{2}$} & \multicolumn{2}{|c|}{ Needle area $/ \mathrm{m}^{2}$} & \multicolumn{2}{|c|}{ Needle area $/ \mathrm{m}^{2}$} & \multicolumn{2}{|c|}{ Needle area $/ \mathrm{m}^{2}$} \\
\hline Fresh & $2.09 \mathrm{~m}^{2}$ & Fresh & $5.13 \mathrm{~m}^{2}$ & Fresh & $5.41 \mathrm{~m}^{2}$ & Fresh & $5.16 \mathrm{~m}^{2}$ & Fresh & $5.11 \mathrm{~m}^{2}$ \\
\hline Dry & $1.89 \mathrm{~m}^{2}$ & Dry & $4.49 \mathrm{~m}^{2}$ & Dry & $4.43 \mathrm{~m}^{2}$ & Dry & $4.38 \mathrm{~m}^{2}$ & Dry & $4.06 \mathrm{~m}^{2}$ \\
\hline \multicolumn{2}{|c|}{$\begin{array}{c}\text { Number of needle } \\
\text { pairs } / \mathrm{m}^{2}\end{array}$} & \multicolumn{2}{|c|}{$\begin{array}{c}\text { Number of needle } \\
\text { pairs } / \mathrm{m}^{2}\end{array}$} & Num & $\begin{array}{l}\text { of needle } \\
/ \mathrm{m}^{2}\end{array}$ & & $\begin{array}{l}\text { f needle } \\
\mathrm{m}^{2}\end{array}$ & & $\begin{array}{l}\text { needle } \\
\mathrm{m}^{2}\end{array}$ \\
\hline & $7 \mathrm{pcs}$ & & pcs & & 3 pcs & & $0 \mathrm{pcs}$ & & 0 pcs \\
\hline
\end{tabular}

Jeseníky plots and compare the averages characterizing individual specimens with the results of other authors. However, this approach is unfeasible in practice because of the complicated growth of dwarf pines. Moreover, the ages of stands (individuals) or size parameters that are mentioned in the literature dealing with dwarf pine biomass are not exactly the same as the ages or size parameters of dwarf pine studied in selected transects. Other sources that mention the biomass of dwarf pine stands (e.g., Malinovskij 1984) cannot be used for comparison as they describe the values of natural communities without specifying size parameters. The dwarf pines in natural communities do not reach the same growth velocity and parameters as those in unnatural (and anthropogenically modified) sites.
The acquired values of the total fresh aboveground biomass converted to 1 ha (135-267 t/ha and 325$482 \mathrm{~m}^{3} / \mathrm{ha}$ ) in stands of the $2^{\text {nd }}$ to the $15^{\text {th }}$ age class may seem high. The reason for the large amount of biomass is the complicacy and the tenacity of pine polycormons. Moreover, the parameters presented in common forestry practice only concern large timber and not the entire aboveground mass. The values of the total aboveground biomass were calculated and are presented intentionally because of the current issue of complete dwarf pine mass removal and the further processing and use of the resulting materials.

The results of this study confirm the assumption that removing the biomass of the dwarf pines would not be an easy task. For example, the aboveground biomass of the oldest stands in the explored area,

Table 4. Basic data from the transect biomass converted to 1 ha

\begin{tabular}{lcccc}
\hline & Transect K1.K2 - average & Transect K3 & Transect V1 & Transect V2 \\
\hline Fresh mass of large timber/ha & $191.625 \mathrm{t}$ & $25 \mathrm{t}$ & $6.5 \mathrm{t}$ & $3.25 \mathrm{t}$ \\
Fresh mass of small timber/ha & $48.25 \mathrm{t}$ & $125.25 \mathrm{t}$ & $108.8 \mathrm{t}$ & $98 \mathrm{t}$ \\
Total weight of fresh mass/ha & $267.25 \mathrm{t}$ & $183.20 \mathrm{t}$ & $148.47 \mathrm{t}$ & $135.32 \mathrm{t}$ \\
Total weight of dry mass/ha & $173.75 \mathrm{t}$ & $119.83 \mathrm{t}$ & $83.86 \mathrm{t}$ & $66.66 \mathrm{t}$ \\
Fresh large timber volume/ha & $250 \mathrm{~m}^{3}$ & $71 \mathrm{~m}^{3}$ & $18 \mathrm{~m}^{3}$ & $6 \mathrm{~m}^{3}$ \\
Fresh small timber volume/ha & $62.5 \mathrm{~m}^{3}$ & $259 \mathrm{~m}^{3}$ & $194 \mathrm{~m}^{3}$ & $153 \mathrm{~m}^{3}$ \\
Total fresh mass volume/ha & $482 \mathrm{~m}^{3}$ & $466 \mathrm{~m}^{3}$ & $388 \mathrm{~m}^{3}$ & $325 \mathrm{~m}^{3}$ \\
\hline
\end{tabular}




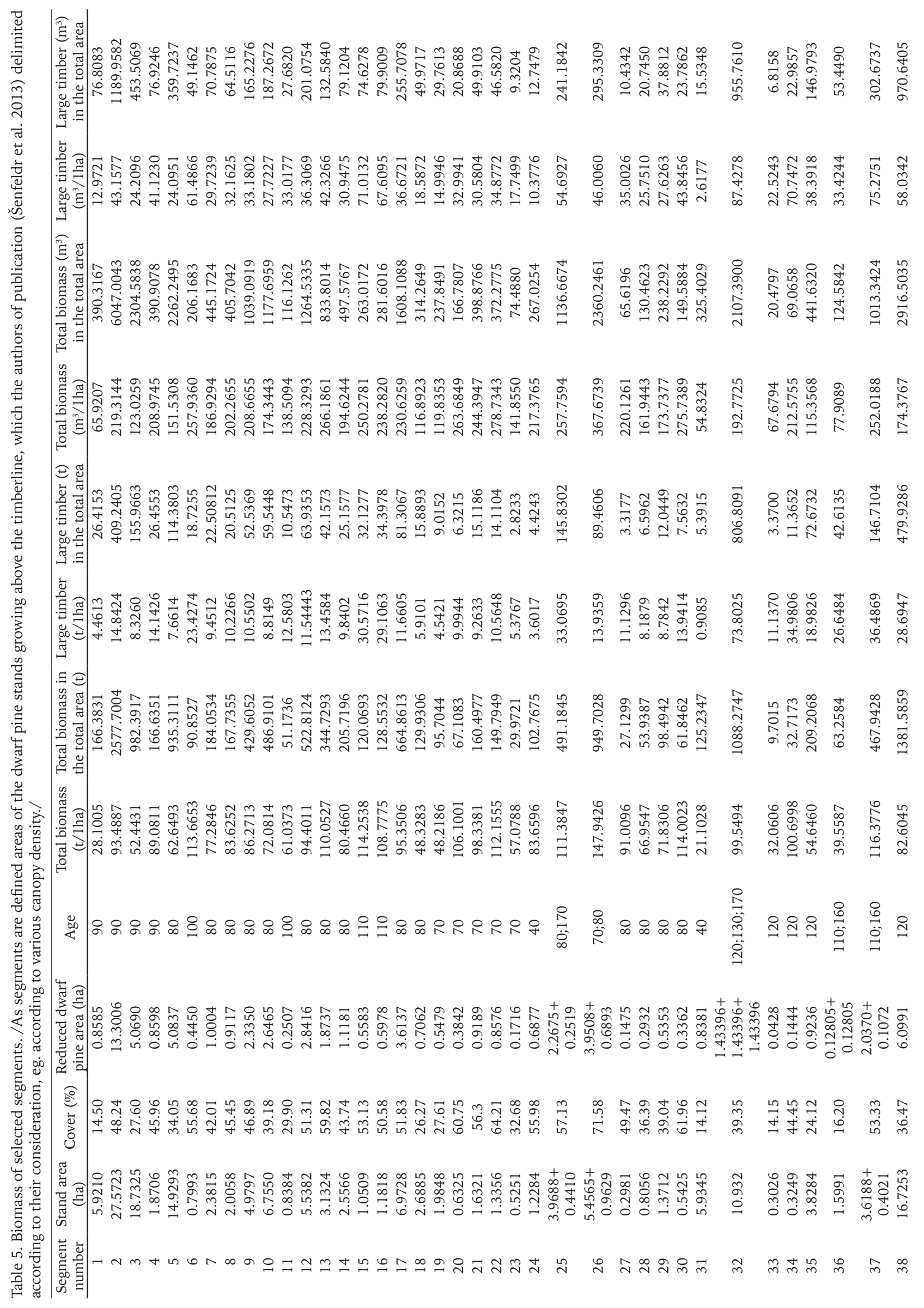




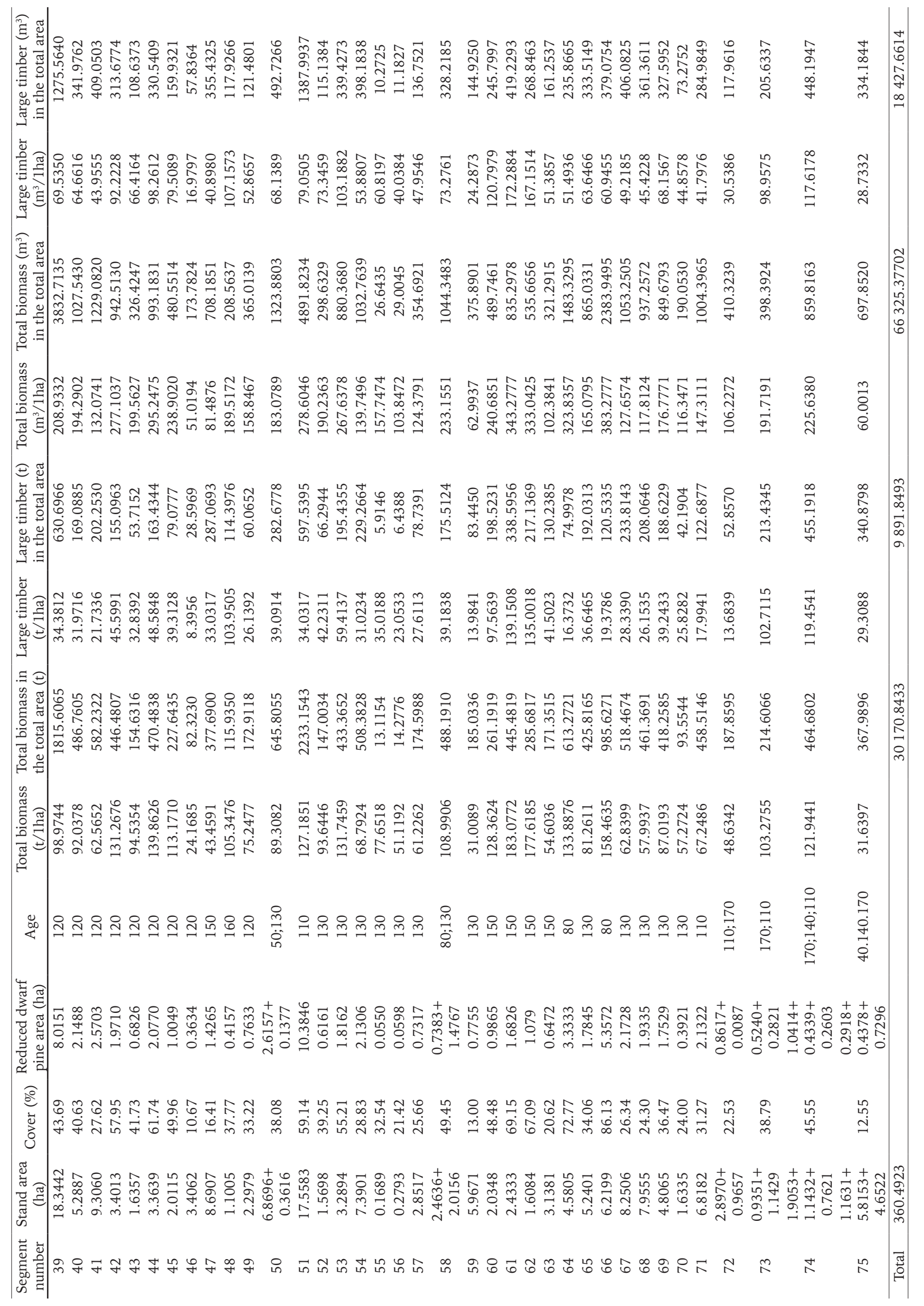


consisting of complicated and tenacious polycormons of the dwarf pine, is comparable to the large timber of a mature spruce stand that is ready to be felled. For example, Corona et al. (2011) mention that in the Czech Republic and other European countries the greatest per-ha deadwood levels are observed in mountain regions. This cannot be explained solely in terms of favorable ecological growing conditions; rather, it is likely linked to the poor accessibility and thus low intensity of forest harvesting. The dwarf pine cutting in these conditions can therefore be problematic due to the complexity of handling and disposal of such quantities of biomass (poor availability of human resources, financial demands, far availability of funds and also legislative context).

Corona et al. (2011) point out that in order to facilitate future research should serve large-scale forest inventories, such as National Forest Inventories. This should expand from traditional variables related to wood and timber production to the assessment of the composition, structure and function of forest ecosystems, and must provide a better understanding of the roles of the components of biological diversity in the provision of multiple forest ecosystem functions. It must result in well-developed partnership among ecologists, nature conservationists, statisticians, resource managers and policymakers (Lindenmayer et al. 2008; Gibbons et al. 2008). Partnerships and compromise among all entities dealing with management of dwarf pine in Hrubý Jeseník Mts. (the Forests of the Czech Republic, State Enterprise, the Administration of the Protected Landscape Area Jeseníky, entities conducting its monitoring and research) in this case are very important.

\section{Conclusion}

Dwarf pines planted on non-indigenous mountainous sites are currently a frequent topic of professional discussions and the summits of the Hrubý Jeseník Mts. in the Czech Republic are no exception. In response to various proposals for dwarf pine reduction, we estimated the amount of aboveground biomass that is located above the timberline in the mountains.

The main result is a methodology for dwarf pine biomass determination that can be used for acquisition and addition of further data, also new trend curves expressing the increasing biomass in relation to age and the creation of equations that could be used to roughly estimate the biomass of all dwarf pine stands, including stands of different age classes and canopy levels on mentioned sites above the timberline. The equations for biomass calculations could also be applied to other mountain ranges where artificially planted dwarf pines of the same seed origin or the same morphological appearance as those existing in the Hrubý Jeseník Mts. are found.

\section{Acknowledgments}

This research was funded by the GS LČR (Effect of Dwarf Pine stands removing on bellow situated stands by upper timber line in Hrubý Jeseník Mts.). Many thanks to Mrs V. Filková for translating of text into English and American Journal Experts for text editing. Also thanks to students of Summer School of Ecology for help with data collecting.

\section{References}

Archibold O.W. 1995. Ecology of world vegetation. Chapman and Hall, London.

Bitterli P. 1987. Jahrringe in Logföhren (Pinus mugo Turra ssp. mugo) als Ausdruck lokaler mechanischer Faktoren. Dendrochronologia 5: 79-95.

Bliss L.C. 1962. Net Primary Production of Tundra Ecosystems. In: Die Stoffproduktion der Pflanzendecke. Vorträge und Diskussionsergebnisse des internationalen ökologischen Symposium in Stuttgart-Hohenheim vom 4.-7. Mai 1960. Lieth H. (ed.). Gustav Fischer Verlag, Stuttgart, pp. 35-46.

Carmel Y., Kadmon R. 1998. Computerized Classification of Mediterranean Vegetation Using Panchromatic Aerial Photographs. Journal of Vegetation Science 9: 445-454.

Corona P. 1987a. Dendrometric and dendrochronological studies on dwarf mountain pine (Pinus mugo). Dendrochronologia 5: 97-103.

Corona P. 1987b. Indagini dendrometriche sul pino montano (Pinus mugo Turra) del Trentino orientale. Dendronatura 8: 48-55.

Corona P., Chirichi G., McRoberts R.E., Winter S., Barbati A. 2011. Contribution of large-scale forest inventories to biodiversity assessment and monitoring. Forest Ecology and Management 262: 2061-2069.

Fišerová D. 1991. Map of actual vegetation of western Krkonoše. Report, manuscript on file. Agency for Nature Conservation and Landscape Protection of the Czech Republic, Prague.

Gibbons P., Zammit Ch., Younggentob K., Possingham H.P., Lindenmayer D.B., Bekessy S., Burgman M., Colyvan M., Considine M., Felton A., Hobbs R.J., Hurley K., McAlpine C., McCarthy M.A., Moore J., Robinson D., Salt D., Wintle B. 2008. Some practical suggestions for improving engagement between researchers policy-makers in natural resource management. Ecological Management \& Restoration 9: 182-186. 
Halounová L. 2004. The automatic classification of b\&w aerial photos. The International Archives of the Photogrammetry, Remote Sensing and Spatial Information Sciences 34: 1-6.

Heikkinen O. 1980. Mountain pine radial growth and the forest limit zone in Gadmental, the Swiss Alps. Fennia - International Journal of Geography 158: 1-14.

Hohl R., Schweingruber F.H., Schiesser H.-H. 2002. Reconstruction of severe hailstorm occurrence with tree rings: a case study in central Switzerland. Tree-Ring Research 58: 11-22.

Holubičková B. 1980. Autochtonní a introdukovaná P. mugo Turra v sudetských pohořích. Opera Corcontica 17: 15-29.

Hošek J., Banaš M., Zeidler M., Kuras T. 2005. Dílčí zpráva z projektu VaV SM/6/70/05 Vliv výsadeb borovice kleče (Pinus mugo) na biotopovou a druhovou diverzitu arkto-alpinské tundry ve Východních Sudetech (CHKO Jeseníky, NPR Králický Sněžník). Návrh managementu těchto porostů. MŽP ČR.

Kadmon R., Harari-Kremer R. 1999. Studying longterm vegetation dynamics using digital processing of historical aerial photographs. Remote Sensing of Environment 68: 164-176.

Kolischuk V.G., Berko J.M. 1967. Growth of Pinus spp. and fluctuations of climate in the Ukrainian Carpathians. Ukrains'kii Botanichnii Zhurnal 24: 39-47.

Kolischuk V.G. 1969. Methods of studying fluctuations in increment of prostrate dwarf forms using Pinus mugo as an example. Ukrains'kii Botanicheskii Zhurnal 52: 852-859.

Kubíček F. 2001. Biomass of the herb and moss layer in forest ecosystems of the Tatra National Park. Ekológia-Bratislava 20: 185-191.

Kubíček F., Šomšák L., Šimonovič V., Majzlanová E., Háberová, I., Rybárska V. 1983. Influence of tourism on dwarf pine (Pinus mugo) communities in the Vysoké Tatry Mountains . Folia Geobotanica 18: 363-387.

Kyncl T., Wild J. 2004. Použití letokruhové analýzy pro datování velkoplošného odumírání kleče. In: Geoekologické problémy Krkonoš. Sborník mezinárodní vědecké konference. Štursa J., Wild J. (eds.). Opera Corcontica 41: 434-440.

Lindenmayer D., Hobbs R.J., Montague-Drake R., Alexandra J., Bennett A., Burgman M., Cale P., Calhoun A., Cramer V., Cullen P., Driscoll D., Fahrig L., Fischer J., Franklin J., Haila Y., Hunter M., Gibbons P., Lake S., Luck G., MacGregor Ch., McIntyre S., Mac Nally R., Manning A., Miller J., Mooney H., Noss R., Possingham H., Saunders D., Schmiegelow F., Scott M., Simberloff D., Sisk T., Tabor G., Walker B., Wiens J., Woinarski J., Zavaleta E. 2008. A checklist for ecological man- agement of landscapes for conservation. Ecology Letters 11: 78-91.

Lokvenc T. 2003. Antropogenní ovlivnění prrírody českých krkonošských jam. Opera Corcontica 40: 287-300.

Lokvenc T., Vacek S. 1991. Vývoj dřevin vysazených na holině a pod porostem rozpadávajícím se vlivem imisí. Lesnictví. 37: 435-456.

Malinovskij K.A. 1984. Digressija biogeocenoticheskogo pokrova na kontakte lesnogo i subal'pijskogo pojasov v chernogore. Haukoba Dumka, Kiev.

Müllerová J. 2005. Use of digital aerial photography for sub-alpine vegetation mapping: A case study from the Krkonoše Mts., Czech Republic. Plant Ecology 175: 259-272.

Packová P., Maděra P. 2004. The natural development of highly productive white willow stands ( $\mathrm{Sa}$ lix alba L.) in the Dyje-Morava alluvium. In: The Economics and Management of High Productivity Plantations. Alvarez J.G. (ed.). University of Santiago de Compostela, Lugo, Spain.

Palombo C., Chirici G., Marchetti M., Tognetti R. 2013. Is land abandonment affecting forest dynamics at high elevation in Mediterranean mountains more than climate change? Plant Biosystems 147: 1-11.

Palombo C., Battipaglia G., Cherubini P., Chirici G., Garfi V., Lasserre B., Lombardi F., Marchetti M., Tognetti R. 2014. Warming-related growth responses at the southern limit distribution of mountain pine (Pinus mugo Turra subsp. mugo). Journal of Vegetation Science 25: 571-583.

Popovic M. 1976. Growth of the mountain pine (Pinus mugo, Turra.) in Yugoslavia. Journal of Biogeography 3: 261-267.

Potočka J. 1999. The dynamics of raised bog vegetation in the Izera mountains. Biology Bulletin of the Russian Academy of Science 26: 540-546.

Šenfeldr M., Maděra P., Buček A., Roštínský P., Špinlerová Z., Culek M., Friedl M., Štykar J., Vavrríček D., Pecháček J., Tippner A., Sedláček A. 2012. Kleč v horské krajině Hrubého Jeseníku. Geobiocenologické spisy. Akademické nakladatelství CERM

Šenfeldr M., Maděra P., Buček A., Roštínský P., Špinlerová Z., Culek M., Friedl M., Vavř́ičck D., Tippner A. 2013. Atlas of expansion and categorization of dwarf pine stands above the forest line in the Hrubý Jeseník Mts. Lesnická Práce, Kostelec nad Černými lesy.

Simon J., Drápela K. 1987. Growth of dwarf pine (Pinus mugo Turra) in the Beskydy and Jeseníky Mountains. Acta Universitatis Agriculturae (Brno)C 56: 141-144. 
Souček J., Lokvenc T., Vacek S., Stursa J. 2001. Site and stand conditions of dwarf pine stands. Opera Corcontica 38: 43-61.

Špinlerová Z., Martinková M. 2006. Growth of mountain pine (Pinus mugo Turra) in relation to the use of other tree species. Journal of Forest Science 52: 217-225.

Špinlerová Z., Martinková M. 2009. Zhodnocení a výběr metod studia agregátu Pinus mugo a př́spěvek $\mathrm{k}$ objasnění jeho role $\mathrm{v}$ imisních horských oblastech. Folia Forestalia Bohemica 7: 88.

Wild J. 2006a. Krummholz and grassland in the summit plateaux of the Krkonoše Mountains: their interactions and long-term dynamics. Doktorská práce, Ústav životního prostředí Přírodovědecké fakulty UK. Praha.
Wild J. 2006b. Vývoj plošného rozšíření klečových porostů - analýza leteckých snímků. In: Dílčí zpráva z projektu VaV SM/6/70/05 Vliv výsadeb borovice kleče (Pinus mugo) na biotopovou a druhovou diverzitu arkto-alpinské tundry ve Východních Sudetech (CHKO Jeseníky, NPR Králický Sněžník). Návrh managementu těchto porostů. Hošek J. (ed.). MŽP ČR, pp. 47-58.

Wild J., Treml V., Potůčková M., Kopalová I. 2007. Vývoj plošného rozšíření klečových porostů analýza historických leteckých snímků. In: Dílčí zpráva z projektu VaV SM/6/70/05 Vliv výsadeb borovice kleče (Pinus mugo) na biotopovou a druhovou diverzitu arkto-alpinské tundry ve Východních Sudetech (CHKO Jeseníky, NPR Králický Sněžník). Návrh managementu těchto porostů. Hošek J. (ed.). MŽP ČR, pp. 88-109. 